\title{
LA INVESTIGACIÓN SOCIAL APLICADA, UNA ALTERNATIVA PARA REDEFINIR LA RELACIÓN SALUD.ENFERMEDAD COMO UN PROCESO SOCIAL*
}

\author{
Sheila Andrea Gómez P*
}

\begin{abstract}
RESUMEN
El presente artículo tiene como objetivo, señalar de manera suscinta la importancia que tiene para el área de salud pública desarrollar investigaciones aplicadas, desde metodologías cualitativas, con el ánimo de entender la relación salud- enfermedad como un proceso social. Para dar cumplimiento a este propósito se hará una breve descripción de la relación que tradicionalmente ha existido entre la investigación y la dimensión salud-enfermedad, señalando la investigación aplicada cómo aquella que permitiría relacionar de manera dialéctica la aparición de la enfermedad y las condiciones estructurales donde se desarrolla, refiririendo la interdisciplinariedad como una estrategia que posibilitaría replantear la pertinencia del paradigma positivista que ha direccionado la investigación en salud y, finalmente dejar expresadas algunas consideraciones reflexivas en torno al tema.
\end{abstract}

Palabras claves: Salud, Enfermedad, Proceso

\begin{abstract}
The following article has the objective of marking concisely the importance of developing applied investigations in the Public Health Sector, from qualitative methodologies, seeking to understand the relation between health-disease as a social process. To achieve this goal I will briefly describe the relation that has traditionally existed between investigation and the area of health-disease, showing applied investigation as the one that permits dialectic relation between the appearance of the disease and the structural conditions in which it develops, identifying interdisciplinarity as a strategy as the one that allows the replanting of the pertinence of the positivist paradigm that has guided investigation in Health and, finally leave some reflexive considerations expressed referent to the theme.
\end{abstract}

Key words: Health, Disease, Process.

* Ensayo elaborado para el seminario Sociedad y Salud en Colombia. Universidad del Valle. 2002

* Estudiante de décimo semestre de Trabajo social, Universidad del Valle. 2004 

pues a través de éste se ha posibilitado construir conocimiento riguroso de la realidad social, dándole peso a las elaboraciones que surgen como resultado de su aplicación, algunas veces de carácter básico y en ocasiones aplicado ${ }^{1}$ dependiendo de los intereses, objetivos y el tipo de conocimiento que se quiera producir.

Concretamente para la elaboración de este escrito, es necesario señalar la relación que ha existido entre la investigación y la dimensión salud- enfermedad, y cómo a través de su utilización se han logrado identificar los diferentes problemas en esta área.

Si bien es cierto las investigaciones en salud no han sido de preferencia abordadas por las ciencias sociales, los profesionales de este ámbito sí estaríamos en capacidad de desarrollarlas siempre y cuando la relación salud-enfermedad se aborde, desde una mirada cualitativa, como un proceso social. La dimensión cualitativa contemplaría dicha relación como un asunto público donde es necesario para dimensionarlo tener en cuenta los aspectos económicos, sociales, políticos y culturales de la sociedad y, cuyo fin sea la búsqueda de las condiciones sociales propicias para la salud y la vida de todas las personas.

En este orden de ideas ¿Cuál seria la importancia de desarrollar investigaciones aplicadas, desde metodologías cualitativas en el área de la salud pública?

Para dar respuesta a este cuestionamiento, empezaré señalando tal como lo hace Ezequiel Ander-egg ${ }^{2}$ - que la característica fundamental de cualquier desarrollo investigativo está enfocada a plantear problemáticas y posibles soluciones, indagando ya sea por inquietudes teóricas o prácticas. Por ello la investigación debe ser una búsqueda sistemática a partir de un marco de referencia conceptual, que utilice instrumentos metodológicos para obtener y verificar los datos, pero adicional a ésto agregaría que el conocimiento que produzca la investigación debe presentar dos características fundamentales: ser producto de un trabajo interdisciplinario y, responder-de acuerdo al contexto- a problemáticas concretas de la realidad específica donde se genere.

Teniendo en cuenta estos dos últimos planteamientos es interesante exponer de modo general, cómo ha sido históricamente la investigación en salud, desde qué enfoques se ha abordado y cuáles los resultados arrojados por ella. Este análisis lo permiten Víctor Mario Estrada y Filiada Ospina ${ }^{3}$, al señalar que la investigación en salud se ha orientado de acuerdo al paradigma positivista, que desde el aspecto biológico ha centrado la investigación se centró en dar explicación unicausal a la enfermedad, focalizando su acción en la búsqueda de estrategias que permitieran desde la curación, a través de la medicalización, dar respuesta a las diferentes situaciones de anormalidad que presentaban las personas. Coherente con este enfoque se consideraban como únicas e iguales las problemáticas en salud, no se hacía diferenciación entre las personas y contextos y el proceso

\footnotetext{
${ }^{1}$ Este tipo de investigación tiene como característica principal señalar situaciones problemáticas y necesidades que requieren ser resueltas de manera práctica en el aquí y en el ahora.

${ }^{2}$ ANDER- EGG. Ezequiel. Técnicas De Investigación Social. Buenos Aires: Magisterio del Río de la Plata, 1993.

${ }^{3}$ Revista PROSPECTIVA \# 1. Replanteamiento de las Políticas de la Salud en los 90. ESTRADA, Víctor Mario Y OSPINA, Filiada. Cali: Universidad del Valle, 1999.
} 
salud- enfermedad sólo se abordaba desde una dimensión cuantitativa, tendencia aún dominante en la investigación en ciencias de la salud, generalizando los episodios de enfermedad y ubicando diagnósticos totalizantes para explicarla; además por que el análisis crítico frente a los resultados arrojados por este tipo de investigación se ha dejado de hacer, reduciéndolo a la simple medición estadística que no permite el abordaje explicativo, sino causal.

Los mismos autores afirman que este tipo de investigación ha logrado dar cuenta de muchos acontecimientos, pero que también otros no han podido ser resueltos desde este enfoque, obligando a la medicina a buscar y abordar las problemáticas en salud con otra óptica, es decir procurando no generalizar los episodios de enfermedad que se presenten, disposición reforzada por la nueva tendencia a ubicar en la multicausalidad la explicación de las enfermedades. Esta mirada permite que se retomen otros escenarios como el social y político, además del físico para dar cuenta de las razones que producen la situación o enfermedad, creando una relación dialéctica entre la aparición de la enfermedad y las condiciones estructurales donde se desarrolla, pues si bien es cierto la materialización de la enfermedad es el signo o síntoma no significa que el elemento que la éste precipitando sea de origen puramente biológico y/o físico; al contrario podría decirse que la ocurrencia de cualquier tipo enfermedad, es producto de una determinada forma de relación social que da paso a la conformación de un sistema social particular, el cual define el papel de cada individuo en él y de esta manera su desarrollo y funciones.

Los anteriores escenarios han sido las tendencias dominantes en la investigación en salud, desarrolladas principalmente por médicos y profesionales designados históricamente para esta labor, quienes desde su saber específico han respondido a las demandas que se les ha hecho en salud, enfocando su conocimiento a ver la relación enfermedad-salud como un estado y no como un proceso social. Esta situación ha limitado significativamente que la medicina pueda responder de manera real y concreta a todas las problemáticas que se presenten en ésta área. $Y$ es justamente en este aspecto donde quiero detenerme para resaltar la importancia de construir un conocimiento relevante para las ciencias de la salud desde la interdisciplinariedad, ya que generalmente la investigación en esta área ha estado dirigida, guiada y alimentada sólo por profesionales de las ciencias médicas, donde se ha desconocido la importancia de vincular otros saberes que permitan desde su especificidad comprender la dimensión estructural de las problemáticas en salud. El trabajo interdisciplinario permite abordar la realidad social desde distintas ópticas y así dar respuestas a las diferentes problemáticas que afectan a las poblaciones, por ejemplo las del área de salud.

Es justamente desde la interdisciplinariedad donde se debe destacar la importancia de realizar un ejercicio investigativo cuya finalidad sea la aplicación, como forma de producir conocimiento en el área de la salud pública.

Al respecto Estrada (1994) da pistas importantes en este sentido, al señalar que ver la salud como un asunto público significa reconocer a los individuos y comunidades no como "agentes" si no como sujetos colectivos, teniendo en cuenta cuál es "el papel que juega la organización socialy económica de la sociedad tanto en la distribución diferencial de la morbilidad como de las condiciones de salud 
de la población" (Estrada; 27). Son por estas consideraciones frente al abordaje de la salud que considero de suma importancia el reconocimiento del saber interdisciplinario, pues sólo si se abordan las dimensiones sociales, económicas, biológicas, culturales y psicológicas, se podrán explicar, analizar y comprender las diferentes manifestaciones de la enfermedad. Además se estarán facilitando determinaciones mucho más completas y precisas sobre la relación saludenfermedad, al abordarla de manera compleja, lo que requiere del mayor entendimiento y precisión de cada variable que la constituye.

Es así que para lograr un abordaje más complejo y completo de la relación salud-enfermedad, la investigación aplicada posibilitaría ver esta relación desde una visión más holística, pues al ser un tipo de investigación cualitativa centra sus lineamientos en el reconocimiento de las realidades a partir de la lectura que los sujetos hagan de ellas, de acuerdo a todas las dimensiones en donde se desarrollen, siendo su principal propósito producir conocimiento concreto para atender situaciones problemáticas del aquí y el ahora. De esta manera la información que esta investigación proporciona se expresa no como un dato estadístico más, sino como unidades semánticas y analíticas, cargadas de sentido que posibilitan el conocimiento y sobre todo la comprensión de diversas realidades a partir de quienes la han construido a través de su propia experiencia en el mundo de la interacción social o parafraseando a Habermas en el mundo de la vida, es decir en aquellas construcciones simbólicas que ponen en juego los constructos objetivos, sociales y subjetivos de cada individuo en un determinado mundo social. $\mathrm{Y}$ es precisamente este mundo de la vida o mundo simbólico el que estamos llamados a comprender los profesionales de cualquier área, no solamente las disciplinas con enfoque social.

Por lo anterior es necesario formular estrategias que posibiliten redefinir metodológicamente la investigación en salud, de tal modo que otras disciplinas como la psicología, la sociología y el Trabajo Social puedan acompañar el proceso de construcción del paradigma positivista en salud, viendo la salud como un producto social que nos incluye a todos.

En este orden de ideas las distintas áreas del conocimiento pueden emprender la labor de redefinir la relación salud-enfermedad, pues sin querer omitir que los discursos contemporáneos han reconocido de alguna forma otras dimensiones, además de la biológica para definir el proceso salud-enfermedad, esta transformación se da de forma paulatina posiblemente, me atrevería a decir, -porque aún no se ha considerado desde los escenarios médicos la necesidad de hacer dicha redefinición; segundo porque la ciencia médica no posee todos los conocimientos sobre las otras variables que definirían el proceso y, finalmente porque quienes tienen ingerencia frente a la formulación e implementación de políticas que tienen que ver con las problemáticas en salud, no muestran voluntad para hacerlo, dificultad de orden estructural que debilita significativamente el avance que pueda tener el cambio en las actitudes y concepciones frente a la salud y la enfermedad. Desde esta perspectiva se siguen considerando las problemáticas en salud desde una mirada sectorizada, la política actual enfatiza la definición de estrategias para aumentar la cobertura, mejorar los tratamientos de medicalización y curación (prevención de la enfermedad), dejando casi de lado la promoción de la vida y la salud y la educación frente a la misma. 
Teniendo en cuenta estos planteamientos es necesario que los profesionales, no sólo del área de salud, se sensibilicen y tomen conciencia de la importancia de integrar sus conocimientos en procesos investigativos cuya finalidad sea la aplicación, pues no pueden seguir sólo abordando las problemáticas en salud desde la medicalización y curación. Es fundamental responder desde la complejidad, lo cual requiere que el proceso salud-enfermedad se defina desde lo colectivo teniendo en consideración todas las variables que influyen en ella:

- Es necesario definir nuevos estilos saludables que permitan ver la salud no como un fin si no como el medio por el cual se logra tener un estado de bienestar y mejorar la calidad de vida, acciones que requieren el reconocimiento de las variables tanto físicas como sociales, económicas y culturales de la población.

- La tarea es empezar a trabajar por la producción de conocimiento socialmente relevante en el área de salud, que logre dar respuesta concreta, real y efectiva a las demandas, contribuyendo a la trasformación de situaciones problemáticas.

- Es fundamental empezar a dimensionar la salud no como un fin si no como un medio que posibilite a través de un proceso continuo riguroso y sistemático el mejoramiento de la calidad de vida de las personas, donde es requisito indiscutible contemplar la dimensión social, económica, política y cultural del proceso.

- Es necesario diferenciar los sectores de la población, buscando reconocer -desde la particularidad- las demandas y posturas frente a la salud; privilegiando en ello el acuerdo a partir de la diferenciación, sólo de esta manera será posible responder de forma real a las diferentes problemáticas de la salud.

Finalmente la investigación aplicada se convierte en una alternativa conveniente para posibilitar la redefinición de la relación salud-enfermedad como un proceso social, pues a través de su ejercicio se podría responder a problemas y situaciones en salud que requieren ser resueltos aquí y ahora.

Así mismo el área de salud podría legitimarse desde lo colectivo, dado que el encuentro de los diferentes saberes, a partir de la interdisciplinariedad, ampliaría la dimensión de las problemáticas en salud y las abordaría desde la perspectiva compleja y completa que se requiere.

\section{REFERNCIAS BIBLIOGRÁFICAS}

ANDER-EGG, EZEQUIEL. Técnicas de investigación social. Buenos Aires: Magisterio del rió de la plata, 1993.

Fundamentos de la saludpública. Tomo I, corporación para investigaciones biológicas. Cáp. 11, participación ciudadana y salud. Medellín, 1997.

Revista Prospectiva \# 1. Replanteamiento de las políticas de la salud en los 90. ESTRADA, VÍCTOR MARIO y OSPINA, Filiada. Cali: Universidad del Valle, 1994.

Recibido en mayo 10 de 2004

Aprobado en junio 15 de 2004 